Article

\title{
A Practical Resource Management Scheme for Cellular Underlaid D2D Networks
}

\author{
Tae-Won Ban ${ }^{\dagger}$ \\ Department of information and communication engineering, Gyeongsang National University, \\ Tongyeong-si 46764, Korea; twban35@gnu.ac.kr; Tel.: +82-55-772-9177 \\ + Current address: Marine Science Bldg. 807, Tongyeong-si 46764, Korea.
}

Received: 27 September 2017; Accepted: 8 October 2017; Published: 13 October 2017

\begin{abstract}
In this paper, we investigate a resource management scheme for cellular underlaid device-to-device (D2D) communications, which are an integral part of mobile caching networks. D2D communications are allowed to share radio resources with cellular communications as long as the generating interference of D2D communications satisfies an interference constraint to secure cellular communications. Contrary to most of the other studies, we propose a distributed resource management scheme for cellular underlaid D2D communications focusing on a practical feasibility. In the proposed scheme, the feedback of channel information is not required because all D2D transmitters use a fixed transmit power and every D2D transmitter determines when to transmit data on its own without centralized control. We analyze the average sum-rates to evaluate the proposed scheme and compare them with optimal values, which can be achieved when a central controller has the perfect entire channel information and the full control of all D2D communications. Our numerical results show that the average sum-rates of the proposed scheme approach the optimal values in low or high signal-to-noise power ratio (SNR) regions. In particular, the proposed scheme achieves almost optimal average sum-rates in the entire SNR values in practical environments.
\end{abstract}

Keywords: D2D; cellular-aided D2D; underlay; mobile caching

\section{Introduction}

Mobile internet traffic has been explosively increasing in recent years [1]. To be specific, multimedia video traffic accounts for about $60 \%$ of total mobile internet traffic and the ratio is expected to grow to $78 \%$ by 2021 [1]. The next generation mobile communication systems requires a much higher capacity to support the explosively increasing multimedia data. It is the easiest way to increase capacity to use wider bandwidth, but radio spectrum, unfortunately, is a limited resource. Many promising technologies such as multiple input and multiple output (MIMO) and small cell systems have been investigated to enhance the spectral efficiency. However, the spectral efficiency is affected by radio channels, which are mainly determined by the distance between transmitters and receivers. This is the reason that higher order modulations can be only applied to devices near base stations (BSs) in current communication systems such as long-term evolution (LTE) and wireless local area network (WLAN). Thus, it is the most effective way to increase the spectral efficiency to reduce the distance between transmitters and receivers rather than other promising technologies.

On the other hand, the quality of service (QoS) for multimedia services is mainly determined by not only transmission rate but also latency, and the latency is closely related to the physical distance between clients and content servers. No matter how much we increase the transmission rate, we can not reduce the latency below a certain level because multimedia data is currently transferred from a content server to mobile clients through many intermediate network entities. If we can shorten the physical distance in end-to-end communications, both the spectral efficiency and the latency will 
be greatly enhanced at the same time [2]. The communication distance can be noticeably reduced by using mobile caching technologies, where multimedia data is cached in mobile devices and thus can be directly transferred to other mobile devices without going through intermediate nodes [3-6]. Device-to-device (D2D) communication is one of the integral parts for the mobile caching networks. Motivated by these contexts, D2D communications have been attracting plenty of interest as one of the promising technologies for the next generation mobile communications systems [7-17]. Furthermore, we can achieve much higher spectral efficiency by cellular underlaid D2D networks, where D2D communications share radio resources with conventional cellular communications as long as the generating interference of D2D communications is regulated to secure cellular communications $[9,10]$.

Despite the extensive previous research on D2D communications, the practical feasibility of cellular underlaid D2D communication networks is not guaranteed because most of them require immoderate intervention of cellular infrastructure such as BS or excessive signalling overhead for channel information feedback. In this paper, we thus investigate a cellular underlaid D2D communication network by focusing on the practical feasibility and we propose a practically feasible resource management scheme for cellular underlaid D2D networks. In the proposed scheme, all D2D transmitters use a fixed transmit power level to remove the signalling for channel information feedback, and each D2D transmitter can determine whether to transmit data on its own without explicit control from BS, while satisfying the interference constraint imposed by cellular networks. The performance of the proposed scheme is evaluated in terms of average sum-rates and the feasibility is also verified in practical environments.

The rest of this paper is organized as follows. In Section 3, system and channel models are described. In Section 4, a practical resource management scheme is proposed. Our numerical results are shown in Section 5. Finally, this paper is concluded in Section 6.

\section{Related Work}

The different transmission modes were introduced for cellular D2D communications [11]: (1) non-orthogonal mode where D2D communications share the same resource as cellular communications, (2) orthogonal mode where D2D communications use dedicated resources, and (3) cellular mode where D2D traffic is transferred through a BS. Based on the three modes, an optimal mode selection scheme was proposed to enhance the performance of D2D communications. While single transmit and receiver antenna are considered in [11], multiple input and multiple output (MIMO) was taken into account by designing pre-coding matrices at each node [12]. In [13], a non-orthogonal centralized D2D communication system was investigated. It was assumed that D2D communications share uplink frequency with cellular communications, as in this paper, and each device can transmit its data in D2D or cellular mode via a BS. An optimal mode selection scheme was proposed to maximize the overall sum-rate. However, the proposed scheme is centralized and can not guarantee the QoS of cellular communications, unlike the proposed scheme in this paper. On the other hand, there have been many studies to investigate power control schemes to deal with cross interference between D2D and cellular communications, which is one of the challenging problems to limit the performance of cellular underlaid D2D communications [14-17]. Optimization problems have been formulated in both non-orthogonal and orthogonal modes and proposed optimal transmit power allocation schemes to maximize the effective capacity based on the formulated optimization problems, while satisfying different delay-QoS requirements [14]. Several suboptimal power control schemes were also proposed and the performance was analysed by simulations. Despite their excellent performance, the power control schemes inevitably yield excessive complexity and signalling overhead. Thus, a distributed power control algorithm was proposed in [15]. The algorithm simply aims to set the individual signal-to-noise and interference ratio (SINR) targets such that the required sum power is minimized with respect to a sum rate target and allocates transmit power levels. MIMO was also considered. However, the simple distributed algorithm can not guarantee the overall performance such as sum-rate and QoS of cellular communications. Contrary to most of the studies considering 
the uplink of cellular networks, the impact of D2D communication on the downlink coverage of a cellular network was investigated in [16]. They developed an analytical model to characterize the coverage probability of cellular networks where a D2D link exists. Shadowing and power control were considered, and BSs and devices were distributed by a Poisson point process. Both centralized and distributed power control schemes were also proposed to maximize the performance of D2D links in terms of sum-rate [17]. However, the QoS of cellular communications was not strictly guaranteed despite their priority over D2D communications.

In spite of many previous studies, to the best of our knowledge, there have been no studies that can strictly guarantee the QoS of cellular communications and provide a moderate level of complexity and signalling overhead for commercialization, which motivated the study in this paper.

\section{System and Channel Models}

In this paper, we investigate a cellular underlaid D2D wireless communication network as depicted in Figure 1. The utilization in cellular uplink is much lower than in downlink because of the asymmetry of mobile internet traffic, and thus it is more efficient for the underlaid D2D network to share uplink resources with cellular communications $[18,19]$. However, the resource sharing between D2D and cellular communications can cause the quality deterioration in conventional cellular communications, which have higher priority over D2D communications. Thus, a cellular infrastructure such as BS imposes an interference constraint on D2D communications to secure the quality of cellular communications. In our system model, we have N D2D pairs, a cellular BS, and a cellular user equipment (UE). In this paper, the association process of D2D pairs and cellular uplink resource scheduling are both beyond the scope of the paper. Thus, we assume that each D2D pair has been already associated. A D2D receiver that wants to receive content is associated with one of the D2D transmitters that cache the wanted content and are located in the proximity of the receiver. In addition, a cellular UE is scheduled to transmit its uplink data to the cellular BS based on a scheduling policy. In this paper, we use a statistical channel model, where $g_{i j}$ denotes the channel coefficient between transmitter $i$ and receiver $j . i \in\{1,2, \cdots, N\}$ or $i=\mathrm{u}$ and $j \in\{1,2, \cdots, N\}$ or $j=\mathrm{b} . i=\mathrm{u}$ denotes a cellular UE and $j=\mathrm{b}$ denotes a cellular BS. We consider a Rayleigh fading model, and thus $\left|g_{i j}\right|^{2}$ is exponentially distributed with mean value $\lambda_{i j}$ [20]. The effect of path loss can be incorporated into $\lambda_{i j}$. Quasi-static block fading is also considered and thus all the channel coefficients are constant during one frame for data transmission and randomly vary each frame. We assume that D2D communications adopt a time division duplex (TDD) scheme and thus the D2D channels are reciprocal without loss of generality. $N_{0}$ denotes the variance of additive white Gaussian noise (AWGN) in D2D receivers. We assume that the transmit power levels of all D2D transmitters and cellular transmitter are fixed at $P$. The cellular transmitter can immediately transmit its data regardless of the presence of D2D communications, while D2D transmissions should be controlled to protect the quality of cellular uplink communications. Thus, a cellular BS imposes an interference constraint on D2D communications. That is, the total interference power that all D2D transmissions cause to the cellular BS should be less than $I_{\text {th }}$ at any moment. $I_{\text {th }}$ is a parameter that the cellular BS can determine by considering both the quality of cellular communications and the performance of D2D communications. Because D2D transmitters use a fixed transmit power, $P_{i}=P$ if D2D transmitter $i$ is allowed to transmit its data; otherwise, $P_{i}=0$. Then, the total interference power received at the cellular BS from all D2D transmitters can be calculated as $\sum_{i=1}^{N} I_{i} P\left|g_{i b}\right|^{2}$, where $I_{i}$ is an indicator function to denote the activity of D2D transmitter $i$, defined as

$$
I_{i}=\left\{\begin{array}{lc}
1, & \text { if D2D transmitter } i \text { transmits data }\left(P_{i}=P\right), \\
0, & \text { otherwise }\left(P_{i}=0\right) .
\end{array}\right.
$$


If we define $\mathcal{I} \triangleq\left\{I_{1}, \cdots, I_{N}\right\}$, D2D communications should comply with the following interference constraint imposed by the BS;

$$
\sum_{i=1}^{N} I_{i} P\left|g_{i b}\right|^{2} \leq I_{\text {th }}
$$

to protect the cellular uplink communications securely. If normalized by $N_{0}$, Equation (2) can be rewritten as

$$
\sum_{i=1}^{N} I_{i} \rho\left|g_{i b}\right|^{2} \leq \frac{I_{\text {th }}}{N_{0}} \triangleq I_{\text {th' }}^{\prime}
$$

where $\rho$ is a transmit power-to-noise ratio of D2D transmitters, hereafter simply called SNR. Then, the signal to noise plus interference (SINR) received at a D2D receiver $i$, denoted by $\gamma_{i}$, can be calculated as

$$
\begin{aligned}
\gamma_{i}(\mathcal{I}) & =\frac{I_{i} P\left|g_{i i}\right|^{2}}{\sum_{k=1, k \neq i}^{N} I_{k} P\left|g_{k i}\right|^{2}+P\left|g_{\mathrm{b} i}\right|^{2}+N_{0}} \\
& =\frac{I_{i} \rho\left|g_{i i}\right|^{2}}{\sum_{k=1, k \neq i}^{N} I_{k} \rho\left|g_{k i}\right|^{2}+\rho\left|g_{\mathrm{b} i}\right|^{2}+1},
\end{aligned}
$$

for a given $\mathcal{I}$. Then, the sum-rate of a cellular underlaid D2D network can be calculated by $\sum_{i=1}^{N} \log _{2}\left(1+\gamma_{i}(\mathcal{I})\right)$. Finally, we need to choose an optimal $\mathcal{I}$ for maximizing the sum-rate while satisfying the interference constraint as follows:

$$
\begin{aligned}
\mathcal{I}^{*}= & \underset{\mathcal{I}}{\arg \max } \sum_{i=1}^{N} \log _{2}\left(1+\gamma_{i}(\mathcal{I})\right) \\
\text { s.t. } & \\
& \sum_{i=1}^{N} I_{i} \rho\left|g_{i b}\right|^{2} \leq I_{\text {th }}^{\prime} .
\end{aligned}
$$

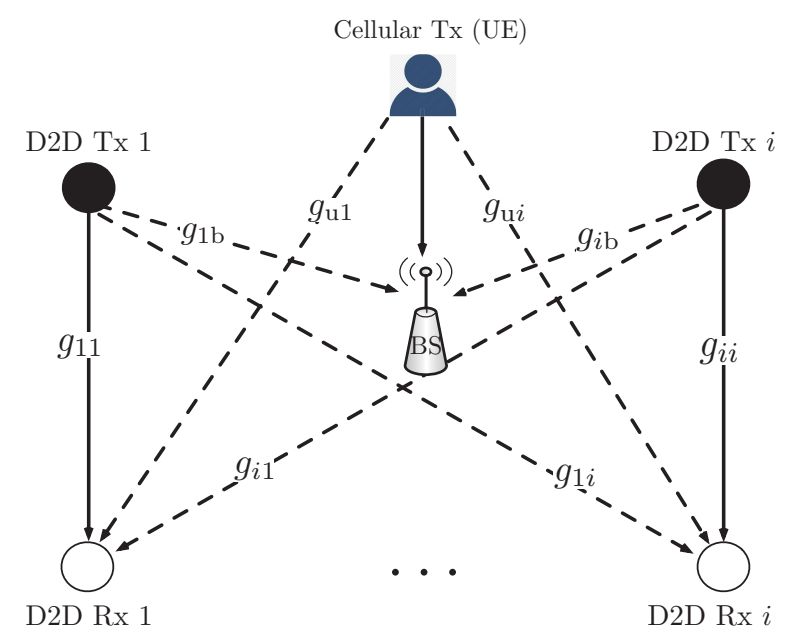

Figure 1. An underlaid device-to-device (D2D) communication network.

\section{Proposed Resource Management for Cellular Underlaid D2D Networks}

The optimization in Equation (5) requires tremendous complexity because it is not a convex problem but a combinatorial problem. In addition, a central node such as a cellular BS should 
have perfect information of entire channels to solve the problem and have full control of D2D communications, which inevitably causes excessive signalling overhead for the feedback of channel state information (CSI). In particular, both the complexity and signalling overhead exponentially increase as the number of D2D pairs $N$ increases. In this paper, we thus propose a fully distributed resource management scheme for cellular underlaid D2D communication networks. The proposed scheme does not require any signalling overhead for CSI and can be operated in a fully distributed manner with a simple signalling broadcast by the BS. Figure 2 shows the flow diagram for our proposed scheme.

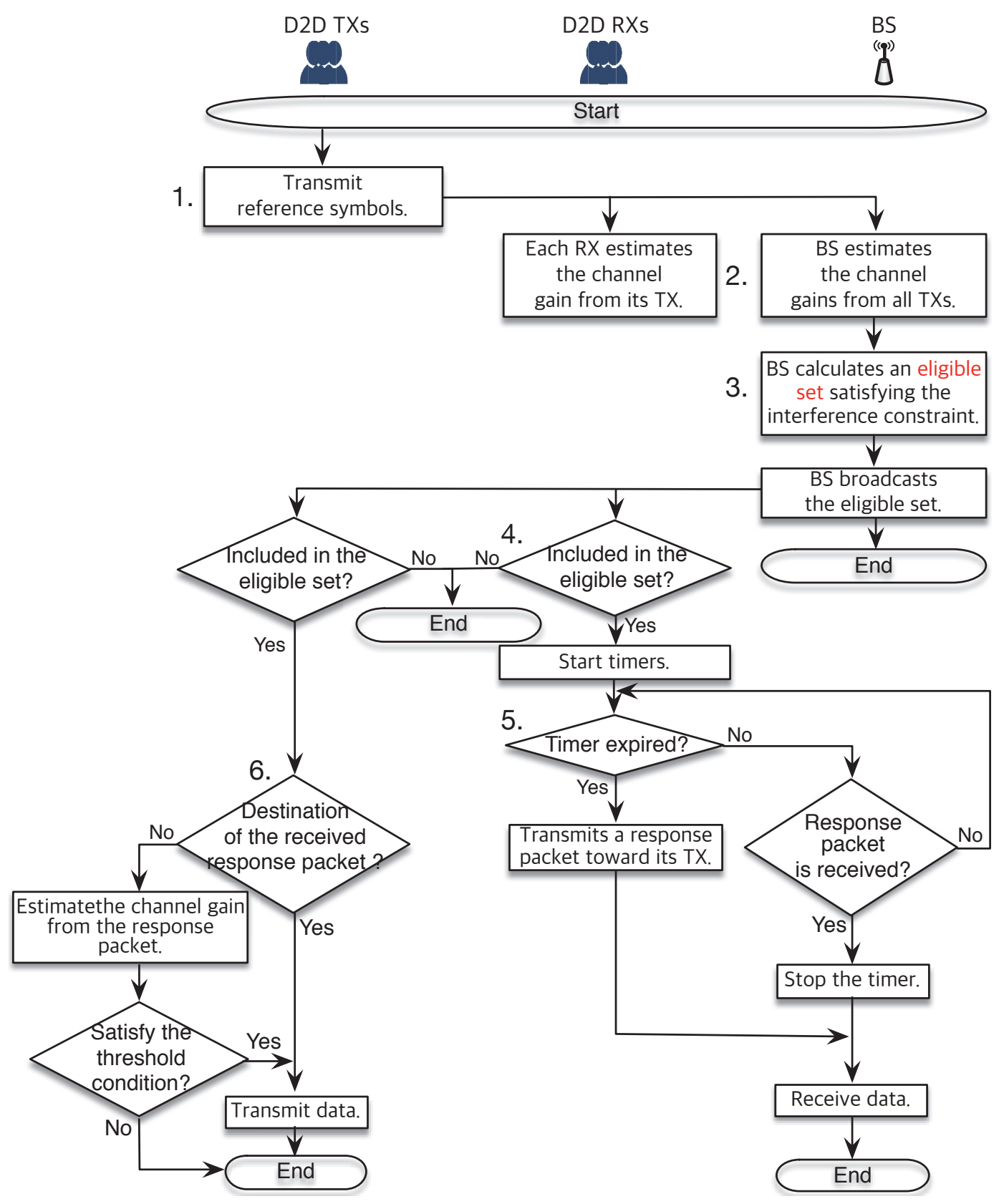

Figure 2. Flow diagram of the proposed scheme for cellular underlaid D2D communication networks.

1. Every D2D transmitter transmits reference symbols to enable D2D receivers and BS to estimate channel gains. We assume that each D2D transmitter is assigned orthogonal radio resource for the reference symbols. 
2. Each D2D receiver only estimates the channel gain received from its associated transmitter, while the BS estimates all channel gains received from all $N$ D2D transmitters.

3. The BS sorts $N$ measured channel gains in an ascending order. The D2D transmitter with the $i$-th largest channel gain is indexed by $\hat{i}$. Then, the sorted set of D2D transmitters can be denoted by $\{\hat{1}, \cdots, \hat{i}, \cdots, \hat{N}\}$ and $\left|g_{\hat{1} \mathrm{~b}}\right|^{2} \leq \cdots \leq\left|g_{\hat{\mathrm{b}}}\right|^{2} \cdots \leq\left|g_{\hat{\mathrm{N} b}}\right|^{2}$ is satisfied. The BS determines an eligible set $\mathcal{E}$, defined by

$$
\mathcal{E}=\left\{\hat{1}, \hat{2}, \cdots, \hat{i}^{*}\right\}
$$

where $\hat{i}^{*}$ can be obtained by

$$
\hat{i}^{*}=\max \left\{\left.\hat{i}\left|\sum_{\hat{k}=\hat{i}}^{\hat{i}}\right| g_{\hat{k} \mathbf{b}}\right|^{2} \leq \mathrm{I}_{\text {th }}\right\} .
$$

Even if all D2D transmitters in the eligible set transmit data simultaneously, the interference constraint imposed by BS is satisfied. Thus, no matter which combination of D2D transmitters in the eligible set transmit data, the interference constraint will be satisfied.

The BS broadcasts a bitmap message with $N$ bits to notify which D2D transmitters are included in the eligible set $\mathcal{E}$.

4. If each D2D transmitter $\hat{i}$ is included in the eligible set, then it waits for a response packet, which will be transmitted from a D2D receiver in Step 5. Otherwise, it terminates this algorithm. If each D2D receiver $\hat{i}$ is included in the eligible set, then it starts a timer. Otherwise, it terminates this algorithm. The value of timer is determined by $\frac{C}{\left|g_{\hat{i} i}\right|^{2}}$, where $C$ is a constant to be determined by a controller and $\left|g_{\hat{\imath}}\right|^{2}$ was measured in Step 2 .

5. A D2D receiver whose timer expires first, denoted by $\hat{t}$, transmits a response packet towards its associated transmitter $\hat{t}$. Note that the timer of the D2D receiver that has the largest channel gain will expire first and thus a D2D pair with the best channel condition can be selected satisfying the interference constraint.

6. All D2D transmitters can receive the response packet transmitted by the receiver $\hat{t}$ in Step 5 . The D2D transmitter $\hat{t}$ can transmit its data, but each D2D transmitter $\hat{i}$ included in $\mathcal{E} \backslash\{\hat{t}\}$ checks the following condition:

$$
\frac{\left|g_{\hat{i} \hat{i}}\right|^{2}}{\left|g_{\hat{i} \hat{i}}\right|^{2}} \geq \eta, \hat{i} \in \mathcal{E} \backslash\{\hat{t}\},
$$

where $\left|g_{\hat{i} \hat{t}}\right|^{2}$ can be obtained by measuring the response packet transmitted by receiver $\hat{t}$, and $\eta$ is a threshold value required in the proposed scheme. All other D2D receivers except $\hat{t}$ stop their timers right after receiving the response packet from $\hat{t}$.

The set of D2D transmitters to transmit data simultaneously can be determined by $\mathcal{T} \triangleq\{\hat{t}\} \cup\left\{\hat{i} \mid \frac{\left|g_{\hat{S}}\right|^{2}}{\left|g_{\hat{q} \hat{t}}\right|^{2}} \geq \eta, \hat{i} \in \mathcal{E} \backslash\{\hat{t}\}\right\}$, satisfying the interference constraint. The D2D pairs with higher channel gain and lower generating interference to the receiver $\hat{t}$ are more likely to be selected to transmit data along with the D2D pair $\hat{t}$. In summary, the proposed scheme is based on a well-known fact that opportunistic resource management schemes can greatly reduce the complexity [21]. Moreover, the proposed scheme selects one user with the highest channel gain out of an eligible set and extra users out of the eligible set satisfying Equation (8) in a decentralized manner to remove most of the complexity and feedback overhead. For a given threshold $\eta$, the SINR of a D2D pair $i$ in $\mathcal{T}$ can be calculated as

$$
\gamma_{i \in \mathcal{T}}^{\text {prop }}(\eta)=\frac{\rho\left|g_{i i}\right|^{2}}{\sum_{k \in(\mathcal{T} \backslash\{i\})} \rho\left|g_{k i}\right|^{2}+\rho\left|g_{\mathrm{b} i}\right|^{2}+1},
$$


and then the sum-rate of the proposed scheme can be obtained as $\sum_{i \in \mathcal{T}} \log _{2}\left(1+\gamma_{i}^{\text {prop }}(\eta)\right)$.

Finally, note that the optimal scheme should carry out $2^{N}$ iterations to calculate sum-rates with the entire channel information feedback at each frame and thus we can not afford the complexity as $N$ increases, while the proposed scheme only requires an $N$-bit message that is broadcast by BS to inform an eligible set.

\section{Numerical Results}

In this section, we evaluate the performance of the proposed scheme in terms of average sum-rate and compare it with optimal performance. In addition, we derive the optimal threshold values to maximize the performance of the proposed scheme. Figure 3 shows the average sum-rates of the proposed scheme where all channels are assumed to be i.i.d. and thus $\lambda_{i j}=1 \forall i$ and $j . N=5$ and $I_{\text {th }}^{\prime}=0$ or $5 \mathrm{~dB}$. For the comparison, it also shows the optimal average sum-rates obtained by solving Equation (5) based on the Brute Force searching algorithm. For a higher $I_{\text {th }}^{\prime}$ denoting that a cellular network tolerates higher interference from a D2D network, the cardinality for the eligible set of D2D transmitters increases and thus average sum-rates of the D2D network also increases due to an increasing gain of user selection diversity. As the SNR of D2D transmitters increases, the average sum-rates of the D2D network also increase due to transmit power gain. However, the SNR values higher than a moderate level reduce the average sum-rate on the contrary because the cardinality for the eligible set of D2D transmitters seriously decreases and thus the user selection diversity decreases as well. The proposed scheme can achieve almost optimal average sum-rates when SNR is low or high, while the average sum-rates of the proposed scheme is lower than the optimal value for moderate SNR values. It should be also noted that the proposed scheme can dramatically reduce the complexity and feedback overhead, compared to an optimal scheme. Figure 4 also shows average sum-rates of the proposed scheme and optimal sum-rates under the same conditions as in Figure 3, except for $N=10$. As $N$ increases, the gain of user selection diversity increases and thus the overall performance is enhanced. The average sum-rates of the proposed and optimal schemes are tabulated and the ratios of average sum-rate obtained by the proposed scheme to optimal value are also summarized in Table 1.

Figures 5 and 6 show the optimal threshold values to maximize the average sum-rates of the proposed scheme with the same parameter values with Figures 3 and 4, respectively. As the SNR of D2D transmitters increases to a moderate level, the optimal threshold value increases and thus $|\mathcal{T}|$ decreases. The optimal threshold value of the proposed scheme decreases if the SNR increases above the moderate level. The excessively high SNR above the moderate level seriously decrease $|\mathcal{E}|$ and thus a low threshold can enhance the performance of the proposed scheme by increasing $|\mathcal{T}|$.

In Figures 7 and 8, we analyze the average sum-rates of the proposed scheme when channels are non-i.i.d. to verify the feasibility of the proposed scheme in practical environments. Table 2 summarizes the average sum-rates of the proposed and optimal schemes and the ratios of average sum-rate obtained by the proposed scheme to optimal value. $(2 \times N)$ D2D nodes are uniformly distributed in a circle with a radius of $200 \mathrm{~m}$ and a cellular BS is located at the center of the circle. The average channel gain between transmitter $i$ and receiver $j$ is determined by $\lambda_{i j}=\min \left(-30 \mathrm{~dB}, L_{i j}^{-4}\right)$, where $-30 \mathrm{~dB}$ and $L_{i j}\left(0 \leq L_{i j} \leq 200\right)$ denote a minimum coupling loss and the distance between transmitter $i$ and receiver $j$, respectively, and $i \in\{1,2, \cdots, N, \mathrm{u}\}, j \in\{1,2, \cdots, N, \mathrm{~b}\}$. It is assumed that the channel bandwidth is $10 \mathrm{MHz}$ and thermal noise power density is $-174 \mathrm{dBm} / \mathrm{Hz}$. The transmit power of all transmitters varies from $0-30 \mathrm{dBm}$ and $I_{\mathrm{th}}=-50$ or $-80 \mathrm{dBm}$. The performance gap between the proposed and optimal schemes is noticeably reduced in the entire SNR region, compared to the i.i.d. channel environments. It is shown that the average sum-rates of the proposed scheme approach the optimal values for entire SNR values and they are almost optimal in a practical region of SNR such as 0-20 dBm. 


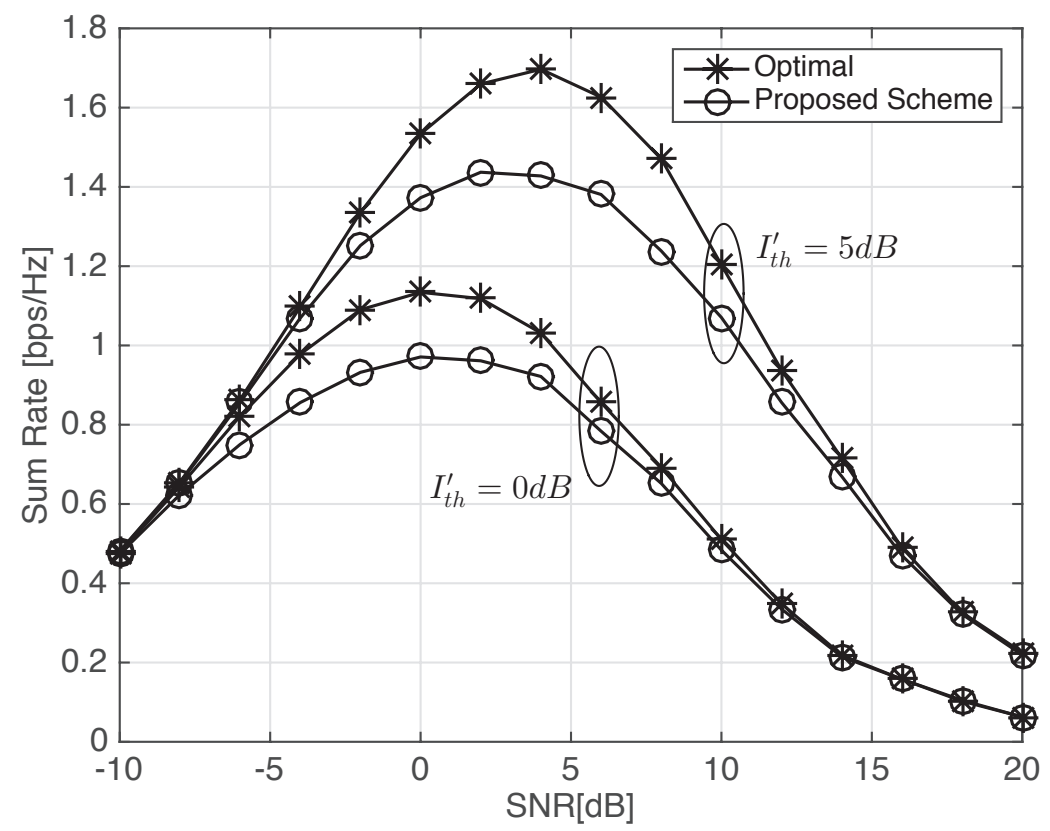

Figure 3. Average sum-rates when all channels are i.i.d., $N=5$, and $I_{\text {th }}^{\prime}=0$ or $5 \mathrm{~dB}$.

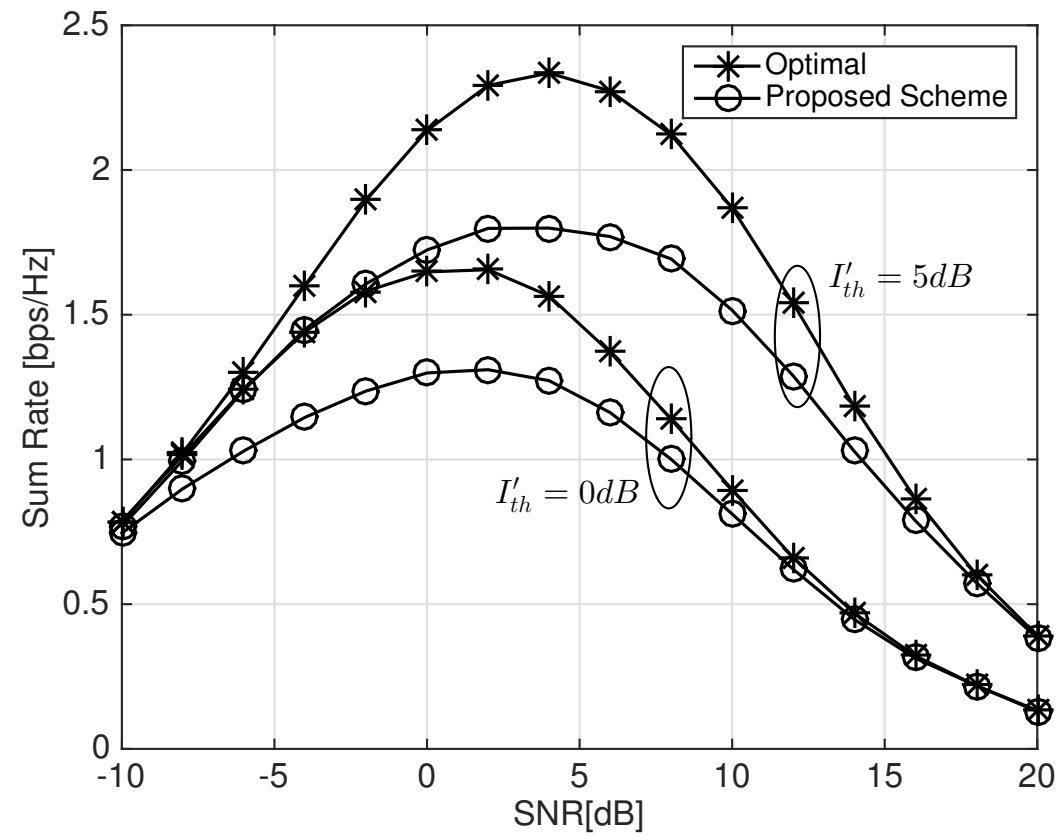

Figure 4. Average sum-rates when all channels are i.i.d., $N=10$, and $I_{\text {th }}^{\prime}=0$ or $5 \mathrm{~dB}$. 
Table 1. Average sum-rates when all channels are i.i.d.

\begin{tabular}{|c|c|c|c|c|c|c|c|c|c|c|c|c|}
\hline \multirow{3}{*}{ SNR (dB) } & \multicolumn{6}{|c|}{$N=5$} & \multicolumn{6}{|c|}{$N=10$} \\
\hline & \multicolumn{3}{|c|}{$I_{\mathrm{th}}^{\prime}=0 \mathrm{~dB}$} & \multicolumn{3}{|c|}{$I_{\mathrm{th}}^{\prime}=5 \mathrm{~dB}$} & \multicolumn{3}{|c|}{$I_{\mathrm{th}}^{\prime}=0 \mathrm{~dB}$} & \multicolumn{3}{|c|}{$I_{\mathrm{th}}^{\prime}=5 \mathrm{~dB}$} \\
\hline & Prop & Opt & Ratio & Prop & Opt & Ratio & Prop & Opt & Ratio & Prop & Opt & Ratio \\
\hline-10 & 0.47 & 0.48 & $99.5 \%$ & 0.48 & 0.48 & $99.8 \%$ & 0.75 & 0.79 & $94.7 \%$ & 0.77 & 0.78 & $98.3 \%$ \\
\hline-6 & 0.75 & 0.82 & $91.3 \%$ & 0.86 & 0.86 & $99.1 \%$ & 1.03 & 1.24 & $82.8 \%$ & 1.24 & 1.30 & $95.3 \%$ \\
\hline-2 & 0.93 & 1.09 & $85.5 \%$ & 1.25 & 1.34 & $93.8 \%$ & 1.24 & 1.58 & $78.2 \%$ & 1.61 & 1.90 & $84.8 \%$ \\
\hline 2 & 0.96 & 1.12 & $86.0 \%$ & 1.44 & 1.66 & $86.6 \%$ & 1.31 & 1.66 & $79.1 \%$ & 1.80 & 2.29 & $78.5 \%$ \\
\hline 6 & 0.78 & 0.86 & $91.6 \%$ & 1.38 & 1.63 & $84.9 \%$ & 1.16 & 1.37 & $84.8 \%$ & 1.77 & 2.27 & $77.9 \%$ \\
\hline 10 & 0.48 & 0.51 & $94.8 \%$ & 1.07 & 1.20 & $89.0 \%$ & 0.81 & 0.89 & $90.7 \%$ & 1.52 & 1.87 & $81.1 \%$ \\
\hline 14 & 0.21 & 0.22 & $98.1 \%$ & 0.67 & 0.72 & $93.6 \%$ & 0.45 & 0.47 & $95.4 \%$ & 1.03 & 1.18 & $86.9 \%$ \\
\hline 18 & 0.10 & 0.10 & $97.8 \%$ & 0.32 & 0.33 & $98.6 \%$ & 0.22 & 0.22 & $99.8 \%$ & 0.57 & 0.60 & $95.5 \%$ \\
\hline
\end{tabular}

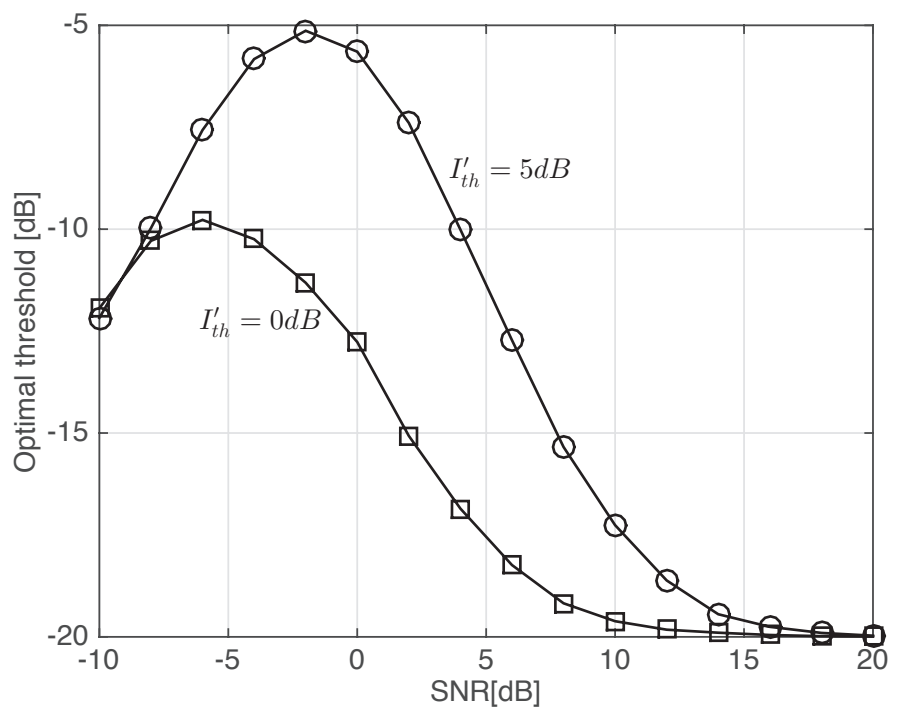

Figure 5. Optimal threshold in the proposed scheme when all channels are i.i.d., $N=5$, and $I_{\text {th }}^{\prime}=0$ or $5 \mathrm{~dB}$.

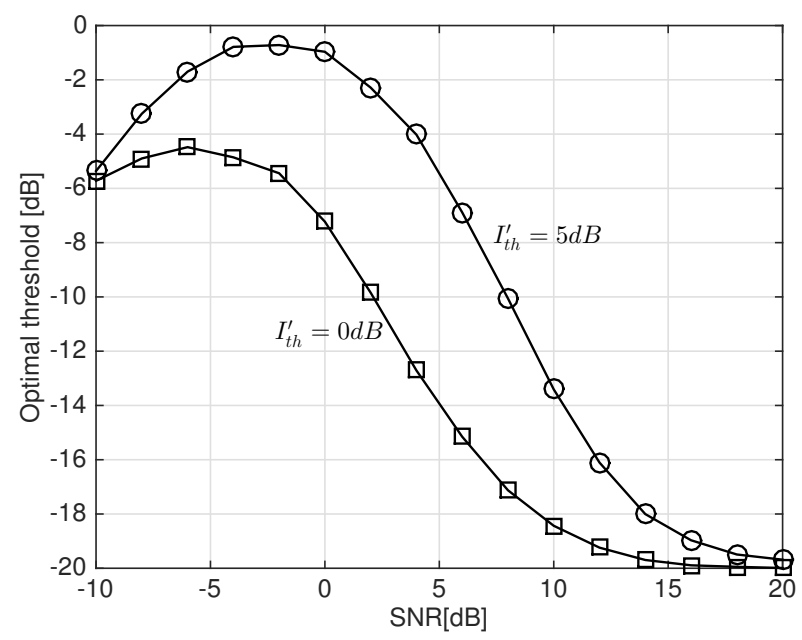

Figure 6. Optimal threshold in the proposed scheme when all channels are i.i.d., $N=10$, and $I_{\text {th }}^{\prime}=0$ or $5 \mathrm{~dB}$. 


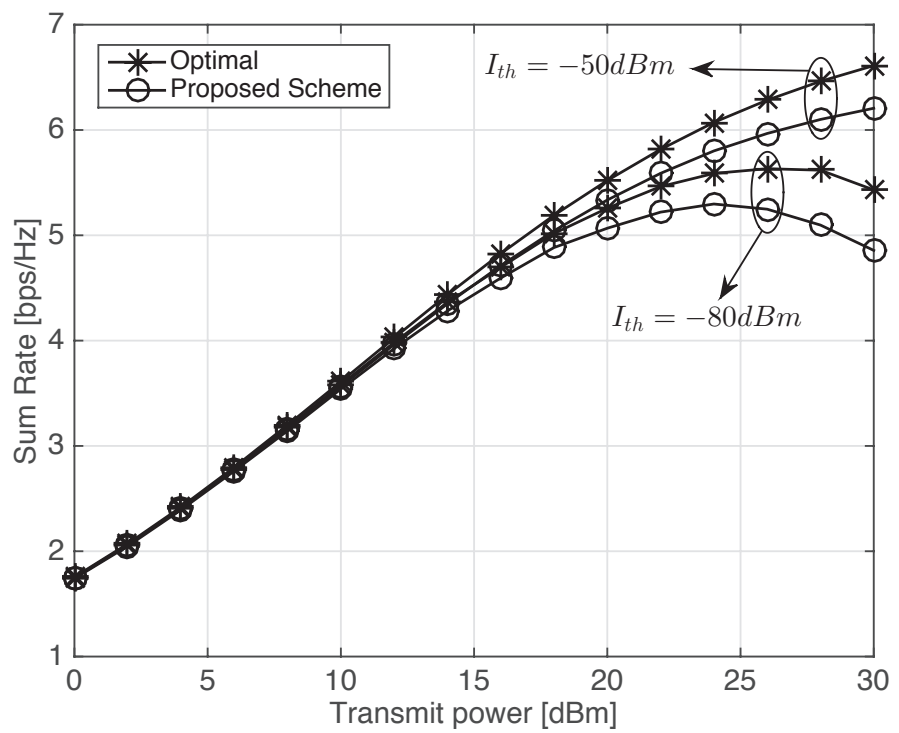

Figure 7. Average sum-rates when all channels are non-i.i.d. and $N=5$.

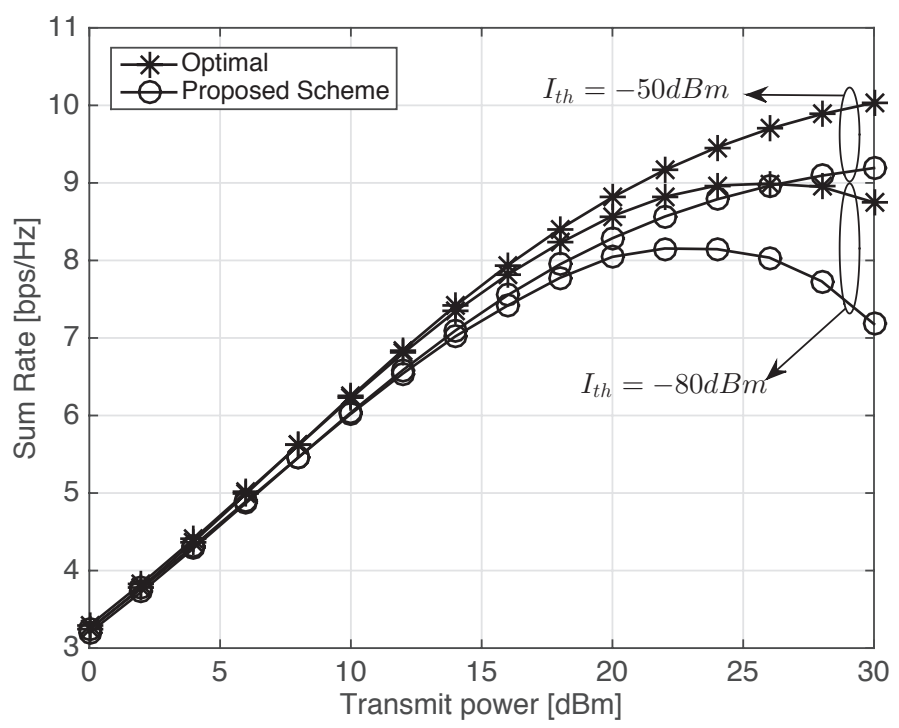

Figure 8. Average sum-rates when all channels are non-i.i.d. and $N=10$.

Table 2. Average sum-rates when all channels are non-i.i.d.

\begin{tabular}{|c|c|c|c|c|c|c|c|c|c|c|c|c|}
\hline \multirow{3}{*}{$P(\mathrm{dBm})$} & \multicolumn{6}{|c|}{$N=5$} & \multicolumn{6}{|c|}{$N=10$} \\
\hline & \multicolumn{3}{|c|}{$I_{\mathrm{th}}=-80 \mathrm{dBm}$} & \multicolumn{3}{|c|}{$I_{\mathrm{th}}=-50 \mathrm{dBm}$} & \multicolumn{3}{|c|}{$I_{\mathrm{th}}=-80 \mathrm{dBm}$} & \multicolumn{3}{|c|}{$I_{\mathrm{th}}=-50 \mathrm{dBm}$} \\
\hline & Prop & Opt & Ratio & Prop & Opt & Ratio & Prop & Opt & Ratio & Prop & Opt & Ratio \\
\hline 0 & 1.74 & 1.75 & $99.7 \%$ & 1.75 & 1.75 & $99.7 \%$ & 3.25 & 3.29 & $98.7 \%$ & 3.20 & 3.24 & $98.8 \%$ \\
\hline 4 & 2.39 & 2.41 & $99.4 \%$ & 2.41 & 2.42 & $99.4 \%$ & 4.32 & 4.41 & $98.0 \%$ & 4.29 & 4.38 & $98.1 \%$ \\
\hline 8 & 3.14 & 3.17 & $99.1 \%$ & 3.17 & 3.20 & $99.0 \%$ & 5.46 & 5.62 & $97.3 \%$ & 5.46 & 5.62 & $97.2 \%$ \\
\hline 12 & 3.92 & 3.98 & $98.5 \%$ & 3.96 & 4.03 & $98.4 \%$ & 6.54 & 6.80 & $96.2 \%$ & 6.59 & 6.85 & $96.2 \%$ \\
\hline 16 & 4.59 & 4.70 & $97.8 \%$ & 4.71 & 4.83 & $97.6 \%$ & 7.42 & 7.82 & $94.9 \%$ & 7.55 & 7.94 & $95.2 \%$ \\
\hline 20 & 5.07 & 5.26 & $96.4 \%$ & 5.34 & 5.52 & $96.6 \%$ & 8.05 & 8.57 & $93.9 \%$ & 8.29 & 8.82 & $94.0 \%$ \\
\hline 24 & 5.30 & 5.59 & $94.8 \%$ & 5.80 & 6.07 & $95.5 \%$ & 8.15 & 8.97 & $90.8 \%$ & 8.79 & 9.46 & $92.9 \%$ \\
\hline 28 & 5.10 & 5.62 & $90.7 \%$ & 6.10 & 6.46 & $94.4 \%$ & 7.73 & 8.95 & $86.4 \%$ & 9.09 & 9.89 & $92.0 \%$ \\
\hline
\end{tabular}




\section{Conclusions}

In this paper, we investigated a cellular underlaid D2D network, which is an enabling technology for mobile caching services. D2D communications are allowed to share radio resources with cellular uplink communications under the assumption that they comply with an interference constraint imposed by a cellular controller such as BS to secure the quality of cellular communications. The performance of D2D communications can be maximized by selecting an optimal combination of D2D pairs to transmit data. However, it causes tremendous complexity of computations and signalling overhead for channel feedbacks to determine an optimal combination complying with the interference constraint. Thus, we proposed a practical resource management scheme for D2D communications. Each D2D pair determines whether to transmit data on its own based on a threshold value and simple bitmap information broadcast by the BS. Thus, the proposed scheme does not require any feedback from the D2D network and does not cause any computational complexity to a BS either. We evaluated the performance of the proposed scheme in terms of average sum-rate and compared it with the optimal scheme. We also derived the optimal threshold values that maximize the average sum-rate of the proposed scheme. Our numerical results showed that the average sum-rates of the proposed scheme approach the optimal sum-rates in low or high SNR regions, despite the tremendous reduction in complexity and signalling overhead. It was also shown that the gap in performance between the proposed and optimal schemes noticeably decreases in the entire SNR region in practical environments where channels are non-i.i.d.

Acknowledgments: This work was supported by the Gyeongsang National University Fund for Professors on Sabbatical Leave, 2017 and Institute for Information \& communications Technology Promotion(IITP) grant funded by the Korea government(MSIT) (No.2015-0-00820, A research on a novel communication system using storage as wireless communication resource).

Conflicts of Interest: The author declares no conflict of interest.

\section{References}

1. Cisco, Visual Networking Index: Global Mobile Data Traffic Forecast Update, 2016-2021, Whitepaper, 2017. Available online: https:/ /www.cisco.com/c/en/us/solutions/collateral/service-provider/visualnetworking-index-vni/mobile-white-paper-c11-520862.html (accessed on 1 October 2017).

2. Caire, G. The Role of Caching in 5G Wireless Networks, Invited Talk in IEEE ICC 2013. Available online: http:/ /icc2013.ieee-icc.org/3_caire_icc-invited-talk-2013.pdf (accessed on 1 October 2017).

3. Cai, X.; Zhang, S.; Zhang, Y. Economic analysis of cache location in mobile network. In Proceedings of the IEEE WCNC, Shanghai, China, 7-10 April 2013; pp. 1243-1248.

4. Wang, X.; Chen, M.; Taleb, T.; Ksentini, A.; Leung, V.C.M. Cache in the air: Exploiting content caching and delivery techniques for $5 \mathrm{G}$ systems. IEEE Commun. Mag. 2014, 52, 131-139.

5. Chae, S.H.; Choi, W. Caching placement in stochastic wireless caching helper networks: Channel selection diversity via caching. IEEE Trans. Wirel. Commun. 2016, 15, 6626-6637.

6. Hong, B.; Choi, W. Optimal storage allocation for wireless cloud caching systems with a limited sum storage capacity. IEEE Trans. Wirel. Commun. 2016, 15, 6010-6021.

7. Giordano, D.; Traversol, S.; Grimaudo, L.; Mellia, M.; Baralis, E.; Tongaonkar, A.; Saha, S. YouLighter: A cognitive approach to unveil YouTube CDN and changes. IEEE Trans. Cogn. Commun. Netw. 2015, 1, 161-174.

8. Son, J. Content Networking Trends: OTT, Global CDN and Operator. Available online: http:/ / www.netmanias.com/en/post/reports/6015/cdn-google-netflix-ott-transparent-cache-youtube / 2013-content-networking-trends-ott-global-cdn-and-operator-cdn (accessed on 1 October 2017).

9. 3GPP TR 22.803 v12.2.0, Feasibility Study for Proximity Services (ProSe) (Release 12). Available online: http:/ / www.tech-invite.com/3m22/tinv-3gpp-22-803.html (accessed on 1 October 2017).

10. 3GPP Work Programme. Available online: http://www.3gpp.org/DynaReport/GanttChart-Level-2.htm\# bm580059 (accessed on 1 October 2017). 
11. Yu, C.-H.; Doppler, K.; Ribeiro, C.; Tirkkonen, O. Resource Sharing Optimization for Device-to-Device Communication Underlaying Cellular Networks. IEEE Trans. Wirel. Commun. 2011, 10, 2752-2763.

12. Morattab, A.; Dziong, Z.; Sohraby, K.; Islam, M. An optimal MIMO mode selection method for D2D transmission in cellular networks. In Proceedings of the IEEE 11th International Conference on Wireless and Mobile Computing, Networking and Communications (WiMob), Abu Dhabi, UAE, 19-21 October 2015; pp. 392-398.

13. Hakola, S.; Chen, T.; Lehtomaki, J.; Koskela, T. Device-To-Device (D2D) Communication in Cellular Network-Performance Analysis of Optimum and Practical Communication Mode Selection. In Proceedings of the IEEE Wireless Communications and Networking Conference (WCNC), Sydney, Australia, 18-21 April 2010; pp. 1-6.

14. Cheng, W.; Zhang, X.; Zhang, H. Optimal Power Allocation With Statistical QoS Provisioning for D2D and Cellular Communications Over Underlaying Wireless Networks. IEEE J. Sel. Areas Commun. 2016, 34, 151-162.

15. Fodor, G.; Reider, N. A Distributed Power Control Scheme for Cellular Network Assisted D2D Communications. In Proceedings of the IEEE Global Telecommunications Conference (GLOBECOM), Houston, TX, USA, 5-9 December 2011; pp. 1-6.

16. Al-Rimawi, A.; Dardari, D. Analytical modeling of D2D communications over cellular networks. In Proceedings of the IEEE International Conference on Communications (ICC), London, UK, 8-12 June 2015; pp. 2117-2122.

17. Lee, N.; Lin, X.; Andrews, J.; Heath, R. Power Control for D2D Underlaid Cellular Networks: Modeling, Algorithms, and Analysis. IEEE J. Sel. Areas Commun. 2015, 33, 1-13.

18. Wang, J.; Zhu, D.; Zhao, C.; Li, J.C.F.; Lei, M. Resource sharing of underlaying device-to-device and uplink cellular communications. IEEE Commun. Lett. 2013, 17, 1148-1151.

19. Ericsson, Frame Structure for D2D-Enabled LTE Carriers. In Proceedings of the Meeting: 3GPP R1-141387, Shenzhen, China, 31 March-4 April 2014.

20. Sklar, B. Rayleigh fading channels in mobile digital communication systems. I. Characterization. IEEE Commun. Mag. 1997, 35, 90-100.

21. Asadi, A.; Mancuso, V. A Survey on Opportunistic Scheduling in Wireless Communications. IEEE Commun. Surv. Tutor. 2013, 15, 1671-1688. 